\title{
HACIA UN MUNDO SIN EMPLEO: MÁS ALLÁ DEL ETERNO CORTO PLAZO
}

\author{
VÍCTOR L. URQUIDI \\ El Colegio de México* \\ In memoriam Redvers Opie
}

Al hacérseme la alta distinción de invitarme a dar la primera conferencia de la
serie "En Memoria del Dr. Opie", que patrocinan el Banco de México y la Cá-
mara de Comercio Británica, deseo ante todo expresar mi agradecimiento al
licenciado Miguel Mancera Aguayo, Director General del Banco, y al señor
Robert Hickman, Presidente de la Cámara, por la oportunidad que se me ha
ofrecido de disertar sobre algún tema de actualidad y que fuera a la vez de in-
terés para la persona a quien se rinde homenaje, el doctor Redvers Opie, falle-
cido en México el mes de febrero pasado. Mas debo también señalar mi especial
reconocimiento al ingeniero Eduardo J. Wygard, no sólo por su vieja amistad
-que compartimos con el doctor Opie-, sino por su interés en los temas del
desarrollo económico y los del corto plazo que, durante su presidencia de la
Cámara de Comercio Británica los años 1981 a 1983 , nos permitieron tanto a
Opie como a mí participar en algunas provechosas discusiones.
Quisiera además referirme a la personalidad y a la trayectoria de Redvers
Opie, pues en cierto modo tuve que ver con su primera visita a México en 1944 ,
que fue el germen de su posterior traslado definitivo a nuestro país y de su in-
tegración a aspectos importantes de nuestra problemática económica. Los que
para 1944 habíamos ya leído, en inglés, la fundamental obra de Joseph Schum-
peter, The Theory of Economic Development, 1 sabíamos que la excelente
* Conferencia en memoria del doctor Redvers Opie patrocinada por el
Banco de México y la Cámara de Comercio Británica, México, D.F., 13 de no-
viembre de 1984 .
1 Schumpeter, Joseph A., The Theory of Economic Development An 
traducción del alemán, hecha en consulta con el autor, y publicada en 1934, llevaba la firma de Redvers Opie. Para los que desconocíamos la lengua original fuera de los balbuceos turísticos ordinarios, el estudio de la traducción de Opie fue fundamental; no salía aún la edición en español que más tarde publicaría el Fondo de Cultura Económica (1944) en versión de Jesús Prados Arrarte. ${ }^{2}$

Cuando llegué a Bretton Woods en julio de 1944, como miembro de la delegación mexicana, a la Conferencia Monetaria Internacional que encabezaba el Secretario de Hacienda y Crédito Público, licenciado Eduardo Suárez, descubrí en la lista provisional de delegados el nombre de Redvers Opie y me propuse trabar contacto con él. Supe entonces que era consejero financiero de la Embajada Británica en Washington y, como tal, asesor importante en las negociaciones que el Reino Unido llevaba a cabo con el gobierno norteamericano acerca de los principales asuntos financieros y económicos que se preveían para la postguerra, entre ellos el arreglo de los créditos de Préstamo y Arrendamiento (Lend-Lease), los saldos bloqueados en libras esterlinas y, sobre todo, la organización de un régimen monetario y financiero internacional estable para la postguerra, en el marco de intereses de lo que entonces se llamaban las $\mathrm{Na}$ ciones Unidas, antes de existir el organismo de tal nombre, es decir, antes de la Conferencia de San Francisco. Dados mis antecedentes de estudio en una universidad británica, no me fue difícil establecer en poco tiempo buena amistad con Redvers y, de paso, conversar con él sobre las cuestiones más significativas de la conferencia. Redvers Opie, por otra parte, había participado sin interrupción en las conversaciones anglonorteamericanas y en las discusiones internas en la Tesorería británica, y se hallaba siempre en contacto con Keynes, jefe de la delegación del Reino Unido -a quien, por cierto, él llamaba "Maynard".

Llevaba yo un encargo especial del Director General del Banco de México, don Eduardo Villaseñor: el de invitar en su nombre a algunos destacados economistas participantes en la Conferencia de Bretton Woods a visitarnos en México para dar conferencias sobre temas de la postguerra, bajo el auspicio del Banco de México. El primero de ellos, a sugestión mía, fue el doctor Opie, quien vino por unos diez días en octubre de 1944. Recuerdo que, además de escuchar sus conferencias, se nos encargó a dos funcionarios del Banco atenderlo y llevarlo a conocer algunos lugares de interés turístico, que en este caso resultaron, inevitablemente, ser Taxco y después Acapulco.

Pocos años más tarde, en mis encuentros con Redvers en Nueva York y en Washington, donde residía habiendo ya dejado de ser funcionario de la Tesorería británica, escuché repetidamente su interés por volver a México, y así lo hizo, entablando en nuestro país lazos académicos, financieros y de negocios, y familiares. Por este motivo nunca dejé de verlo y de intercambiar con él ( sin estar siempre de acuerdo) ideas y opiniones sobre la situación económica internacional, la de América Latina y en particular la de México, a la que él se dedicaría profesionalmente más tarde.

Así pues, son cuarenta años de recuerdos de amistad e intercambio profe-

Inquiry into Profits, Capital, Credit, Interest, and the Business Cycle. Translated from the German by Redvers Opie. Cambridge, Massachusetts, Harvard University Press, 1934.

2 Schumpeter, Joseph A., Teoría del desenvolvimiento económico. Una investigación sobre ganancias, capital, crédito, interés y ciclo económico. Traducción de Jesús Prados Arrarte, México, Fondo de Cultura Económica, 1944. 
sional con Redvers Opie los que hace unas semanas me animaron a abordar el tema que hoy ofrezco en respuesta a la invitación que he mencionado. Pero hay más: tuve oportunidad, hace apenas dos semanas, con motivo de una reunión académica en Oxford, de pisar el suelo y recorrer los jardines del College de esa ciudad del que fue Fellow nuestro homenajeado: Magdalen College (que Redvers me enseñó a pronunciar como se debe: "Maudlin" - como también lo subraya, para los extranjeros no entendidos de las especialidades de la dicción británica del inglés, una reciente guía de los colegios de Oxford). Creo no haber tenido ocasión anterior de visitar el College del que fue distinguido miembro el doctor Opie, y si no es considerado como el más hermoso de los edificios universitarios de Oxford, es de los más antiguos y cuenta con un extraordinario claustro, una capilla construida en el siglo XV y extensos y bellos jardines. Debe haber sido un lugar ideal para el joven Redvers cuando iniciaba su carrera como economista, no sólo en la enseñanza y la investigación propias de la tarea académica, sino también en el conocimiento del mundo ajeno: en los años veinte y treinta Redvers visitó la Unión Soviética, Alemania y otros países del "Continente" (como lo llaman los británicos) y los Estados Unidos y Canadá. Hizo su doctorado en la Universidad Harvard, donde el profesor Frank Taussig, su maestro, lo presentó a Schumpeter con recomendación de que éste le encargara la traducción de su libro. A sus conocimientos de alemán, francés y ruso, Opie habría de añadir, una vez establecido en México, una capacidad notable de manejo de la lengua española - sin perder su tono británico de base.

No me extiendo más en reminiscencias, pero me pareció importante citar estos antecedentes, porque el doctor Opie, conocido en México al final de sus días, y a ya avanzada edad, por su edición trimestral de un sesudo y agudo análisis de la coyuntura económica mexicana y por su labor de asesoría a las Cámaras Británica y Mexicano-Norteamericana de Comercio, tuvo siempre preocupaciones simultáneas por la situación y la perspectiva a corto plazo y por las tendencias de desarrollo a plazos mediano y largo. No en balde fue el traductor de Schumpeter. Intentaré, a continuación, llevar como marco de fondo estas perspectivas tanto de plazo largo como de lo que me he permitido llamar el "eterno" corto plazo.

Mis observaciones serán de carácter general -con alguna referencia final al caso de México-, situadas en un plazo que delimita, en primera instancia, el fin del presente siglo, distante apenas quince años, o sea en 1999, pero en el contexto de un intento de ver más allá. Pues todos los elementos del desarrollo que conocemos, y en particular la población, la tecnología y la organización de la sociedad nacional y de la economía internacional seguirán estando presentes, en algunos casos con dinamismos y retroalimentaciones más complejos, en ese futuro más lejano que tendrán que vivir los 7 a 8 mil millones de habitantes que las Naciones Unidas proyectan para el mundo en su conjunto hacia el año 2025. En un planeta crecientemente interdependiente en lo económico, en lo político y en lo ecológico, es posible que los problemas que tengan que afrontar los 1300 a 1400 millones de personas que para entonces pueblen los territorios hoy llama- 
dos "desarrollados" no sean radicalmente distintos a los que afecten a los 6000 a 6700 millones que se prevé estarán asentados en las áreas hoy denominadas "países en vía de desarrollo". 3 Por lo menos tendrían en común los pueblos del Norte y del Sur el tener que vivir en gran medida sin empleo.

Sin incurrir en ningún determinismo poblacional, ya que intervienen tantos otros factores e incidencias, vale la pena detenerse en algunas magnitudes demográficas destacadas -siquiera por la sencilla razón de que se supone que entre el 30 y el 50 por ciento de la población total, según los países y las regiones, debiera formar parte de la fuerza de trabajo. Esta Población Económicamente Activa (PEA), deberá contar con un ingreso adecuado, en dinero y en especie, lo que supone a su vez que deberá tener empleo, remunerado o no. La alternativa será, por supuesto, estar en condición de desempleo, también remunerado o no. Y habrá sin duda muchas modalidades intermedias que más adelante procuraré esbozar.

Las primeras proyecciones demográficas en que hay que fijar la atención son las referentes al año 2000. Siguiendo supuestos adoptados por los técnicos de las Naciones Unidas, puede preverse que la población mundial a fin de siglo será de unos 6100 millones, de los que corresponderán a los países desarrollados unos 1250 millones y a los países en desarrollo entre 4700 y 4800 . Dadas las tasas diferenciales de fecundidad (en lo principal), los incrementos entre 1984 y el año 2000 serán de cerca de 1400 millones en el mundo en su conjunto; de éstos, menos de 150 millones en los países del primer grupo y el resto, alrededor de 1250 millones, en los del llamado Tercer Mundo. Entre estos últimos países, por regiones, los principales incrementos ocurrirán en Asia meridional (535 millones), en África (340 millones) y en Asia Oriental (230 millones). En América Latina se estima que el aumento será de unos 150 millones.

Traduciendo estas cifras a tasas medias anuales, las más elevadas son las de África, de poco más de 3\% anual en el mismo periodo, seguidas de las de América Latina, del 2.5\%, las de Asia Meridional, del

\footnotetext{
3 Proyecciones establecidas por las Naciones Unidas para la Conferencia Internacional de Población, México, agosto de 1984. Véase el documento E/CONF. 76/PC/40, Review and Appraisal of the World Population Plan of Action-Report of the Secretary General, 10. de diciembre de 1983, especialmente los párrafos 115 a 130 y el cuadro 1 . Las cifras que se citan corresponden a las proyecciones llamadas "intermedia" y "baja", es decir, que suponen descensos de la fecundidad. Para simplificar, se aproximan en este texto a centenas de millones.
} 
$2.2 \%$, y las de Asia Oriental, del 1.14\%. En contraste, las proyecciones relativas a las poblaciones de los países desarrollados indican, entre 1984 y el año 2000, tasas medias anuales de alrededor de $0.5 \%$ en su conjunto, con crecimientos muy lentos, del $0.3 \%$ en Europa. ${ }^{4}$

El Banco Mundial estima cifras semejantes y, al separar por grupos de países y por nivel medio de ingreso real, proyecta tasas de incremento demográfico que van desde $2.9 \%$ anual para los de más bajo ingreso hasta $2.1 \%$ anual entre los países en desarrollo de nivel mediano exportadores de petróleo y $1.3 \%$ en China e India. Respecto a los países industrializados, coincide con las estimaciones de $\mathrm{Na}$ ciones Unidas. Son todos estos promedios por grupos de países, y por supuesto las cifras varían de país a país, a veces considerablemente. 5

Lo que interesa señalar con mayor precisión es la perspectiva de la fuerza de trabajo para el año 2000. Según los análisis del Banco Mundial, la proporción de la población total constituida por aquella en edad de trabajar ( 15 a 64 años) no varía gran cosa entre las regiones, aunque la estructura por edades sea bastante distinta. En los países de menor nivel de ingresos, va del 50 al 60 por ciento, y en los industrializados oscila entre el 58 y 66 por ciento. 6 En la actualidad la fuerza de trabajo total -la parte de esa población que tiene actividad económica (PEA) - es de alrededor de 1300 millones en los países en desarrollo y de 530 millones en los desarrollados. Suponiendo en los primeros una tasa media de desempleo abierto de $15 \%$ y en los segundos de 12\% (y sin contar el subempleo ni el desempleo disfrazado de empleo informal), la población empleada hoy es de unos 1100 millones en los países del Tercer Mundo y de 640 millones en los del llamado "Norte".

La perspectiva de incremento de la fuerza de trabajo o PEA difiere considerablemente entre los dos grupos de países. Para fin de siglo, la PEA crecerá mucho más rápidamente en los países en desarrollo, más o menos en correspondencia con su tasa de incremento demográfico, aun cuando con variantes que dependerán de la estructura por edades. En los países de elevada fecundidad y que prevean descensos de la mortalidad, la tasa de incremento de la fuerza de trabajo será superior, durante plazos de 10 a 15 años, a la tasa de incremento general de la población. En muchos, según los

4 Ibid., cuadro 1.

5 Banco Mundial, Informe sobre el desarrollo mundial, 1984. Washington julio de 1984, cuadro 1, Proyecciones demográficas, pp. 222-223 y cuadro 19, Crecimiento y proyecciones de la población, pp. 286-287.

6 Ibid., cuadro 21 , pp. 290-291. 
niveles culturales y las estructuras económica y social, los incrementos de la PEA incluirán, en proporción creciente, el concurso de la mano de obra femenina, ya sea o no remunerada, con tendencia a ingresar al trabajo remunerado; asimismo, el descenso previsible de la mortalidad hará que permanezcan en la PEA mayores contingentes absolutos de trabajadores. La PEA efectiva de los países en desarrollo incluye siempre, además, una parte de la población menor de 15 años.

Como quiera que sea, y suponiendo que en los países desarrollados la PEA se mantendría a un nivel de $45 \%$ de la población total y en los paises en desarrollo en una proporción de $35 \%$, entrarán al mercado de trabajo entre 1984 y el año 2000 unos 67 millones de personas en los primeros, y unos 440 en los segundos. 0 sea que habría que dar trabajo a poco más de 500 millones de personas adicionales de aquí al año 2000 . Y si se quisiera absorber el desempleo abierto actual, reduciéndolo a no más del $6 \%$ de la PEA, habría que añadir otros 150 millones al empleo, de los cuales casi 120 en los países en desarrollo y unos 30 en los desarrollados. Sumada la reducción del desempleo al impacto del incremento de la fuerza de trabajo, los sistemas económicos del mundo necesitarían crear 650 millones de empleos en 15 años, correspondiendo 560 a los países en desarrollo y 90 a los países desarrollados, para llegar a una situación de elevado nivel de empleo -es decir-con desempleo de sólo $6 \%$ en su conjunto.

Por supuesto que la tarea será gigantesca. En las regiones de mayor incremento relativo y absoluto de la fuerza de trabajo -en especial en Asia Oriental y Meridional y en África-, será mayor todavía. En América Latina las cifras absolutas serán menores, pero la tasa de incremento del empleo que se requerirá será apenas inferior a la de África.

Salvo que ocurran cambios verdaderamente dramáticos en las tasas de fecundidad y mortalidad, y en las tasas de participación en la fuerza de trabajo, lo anterior presenta un cuadro general que no induce a ser demasiado optimista sobre las tendencias del empleo, a la luz de la experiencia de expansión económica mundial de los últimos quince años. Considerando sólo las perspectivas del comercio internacional, éste tendría que crecer a tasas muy superiores a las actuales para dejar a los países en desarrollo un margen de ahorro propio para crear el empleo necesario. Y entran en juego muchos otros factores que se mencionarán brevemente más adelante.

Antes, vale la pena asomarse un poco al siglo XXI, con proyecrinnes al año 2025 (de las que se dispone respecto a población). Sin 
entrar en pormenores, entre el año 2000 y el año 2025 los países en desarrollo, a menos de que su tasa de incremento demográfico descienda en forma aún no imaginada, tendrán que crear otros $400 \mathrm{mi}$ llones de empleos. En cambio, los países desarrollados, por falta de mano de obra propia, verían disminuir el número de puestos de trabajo en unos 15 millones o tendrían que llenar cualquier posible demanda adicional con inmigrantes provenientes del llamado Sur, o bien intensificar la automatización. Todo esto en el supuesto de que el desempleo abierto después del año 2000 se mantuviera en un 6\% de la PEA. No tendría gran consistencia el especular sobre otro tipo de cifras entre el año 2000 y el 2025 , sobre todo en materia económica, pero los datos demográficos y como derivados de ellos los montos de la fuerza de trabajo disponible, tendrán los órdenes de magnitud indicados.

En resumen, de aquí al año 2025 -y siempre con desempleo abierto no superior al $6 \%$ - los países en desarrollo deberán crear empleo para casi 1000 millones de personas, de los cuales 560 para el año 1999. Los desarrollados requerirán crear unos 75 millones para 2025, pero, antes, deberán incrementar el empleo en 90 millones para el año 1999. Bajo ciertos supuestos los 75 millones al año 2025 podrían complementarse a partir del año 2000 con unos 15 millones de trabajadores incorporados del actual Tercer Mundo, para mantener empleo constante en el Norte.

Ahora bien, nada indica por ahora que puedan lograrse todas estas metas para dentro de 15 años ni, mucho menos, para el año 2025. Desde principios de los años setenta, los países altamente industrializados, tanto de economía de mercado como de planificación central, han mostrado claros indicios de crecimiento más lento y aún han caído en una prolongada depresión. Cierto es que la economía norteamericana ha registrado tasas elevadas de crecimiento en algunos trimestres recientes, algunos inusitadamente dinámicos. Sin embargo, las proyecciones a mediano plazo que se conocen no hacen pensar en una expansión capaz de reducir los actuales niveles de desempleo, estancados en alrededor del $7 \%$ de la fuerza de trabajo disponible. Las economías de los países de Europa Occidental ofrecen horizontes poco definidos y en todo caso no muy altos. El Japón, por razón de su singular combinación de tecnología avanzada y comercialización audaz, mantiene tasas de crecimiento sostenidas, con bajo desempleo (que obedece en parte a modalidades institucionales de vieja raíz).

Los países en desarrollo, en su conjunto, todavía no salen airosos de las crisis económica y financiera iniciadas en 1981 y, aunque algu- 
nos en Asia Meridional y Sudoriental acusan dinamismo, las perspectivas a mediano plazo de esas subregiones no están garantizadas ni tienen mucho peso en la economía mundial. Son además países que figuran entre los de mayor población en el mundo, algunos con tasas de incremento rápidas, lo que ejerce fuerte presión sobre las posibilidades futuras de empleo.

Entre los países en desarrollo merecen especial atención aquellos de reciente y pujante industrialización, los llamados NICs en la jerga económica internacional moderna, comprendidos cinco latinoamericanos, ocho asiáticos y unos cuantos más proyectados a esa etapa si fructifican sus planes de desarrollo. Podrán tal vez alcanzar considerable dinamismo, pero tal vez no lo suficiente, en su conjunto, para absorber adecuadamente en empleo productivo sus incrementos de PEA. La industrialización previsible en otras áreas, notablemente África Subsahariana, Centroamérica y el Caribe, varios otros países de América Latina y del Pacífico, difícilmente podrá ser suficiente para dar trabajo productivo a su población futura que lo necesitará.

Aun en el caso de los países $N I C$, que han incorporado tecnología moderna y poseen ventajas de estructura salarial para la exportación de manufacturas, la perspectiva pudiera verse frustrada por el auge del proteccionismo en los grandes mercados de los países altamente industrializados. No se avecina una tendencia por parte de éstos de mayor apertura a importaciones del Tercer Mundo mientras la expansión económica de este norte sea relativamente modesta, vista a fines de siglo.

Supongamos, sin embargo, en ausencia de otros factores, que este tipo de previsión fuera muy poco alentador. ¿Cuáles serían esos otros factores? Destaca entre ellos el tecnológico, que históricamente ha tendido a economizar mano de obra, especialmente la de menor calificación, tanto en la industria como en la actividad primaria y los servicios. La tecnología ha elevado la productividad, así como el ingreso real de los trabajadores, pero casi siempre ha tendido a desplazar empleo en las unidades productivas de las ramas concretas de actividad en que ha sido aplicada. Su efecto indirecto ha sido sin duda positivo, pero con la automatización y su forma acentuada reciente de incorporación de la microelectrónica a una gran variedad de procesos de manufactura, producción agropecuaria y servicios de apoyo a la industria y al comercio, los efectos positivos en el empleo global empiezan a ponerse en duda. Inclusive el llamado "redespliegue industrial" de los países del norte hacia el sur pudiera, en determinadas circunstancias, revertirse con base en microelectrónica y en robotiza- 
ción a favor de los países de alta industrialización. ${ }^{7}$ No debe descuidarse el hecho de que se trata de una de las áreas de tecnología avanzada en que los países desarrollados llevan gran delantera a los países en desarrollo - distancia difícil de reducir dado el escaso esfuerzo de investigación tecnológica en la mayoría de los países en desarrollo, sin el cual tampoco es posible transferir y absorber nuevas tecnologías.

A la microelectrónica debe agregarse otro de los grandes campos de adelanto, el de la biotecnología, resultado de concentrada investigación científica, aplicable a la agricultura y a la industria, por ahora a favor de los países ya industrializados. Las ramas de avance en biotecnología coinciden en lo general en la tendencia a economizar mano de obra calificada en las unidades de producción.

La mayor parte de la investigación tecnológica llevada a cabo en el norte, y buena parte de la poca realizada en el sur, llevan una fuerte carga anti-empleo, en el sentido social de éste. En tanto se pueda hablar de un patrón de investigación tecnológica, se caracteriza por la búsqueda de mayor productividad por obrero ocupado y por economía en el uso de mano de obra. Los países en desarrollo suelen copiar este patrón; si no lo hacen, no adquieren competitividad internacional. Pero el costo en capital y en adquisición de tecnología les puede resultar ruinoso, o les puede acentuar el desempleo o el subempleo, o todo junto.

Desde hace unos quince años se viene insistiendo a nivel de Naciones Unidas, y crecientemente entre grupos privados de los países desarrollados y en muchos de los del sur, en las alternativas tecnológicas que signifiquen, entre otras cosas, mayor empleo de mano de obra por unidad de producción física, pero con alto rendimiento productivo. Las llamadas tecnologías intermedias nacen en parte de la tecnología tradicional de los países del Tercer Mundo y se presentan, a veces exageradamente, como opuestas a las tecnologías avanzadas. Se pensó alguna vez que, aun combinadas las dos - como lo expresa la política china de "caminar sobre dos piernas"- se podría lograr contrarrestar el efecto desplazador de empleo de la tecnología industrial de punta. Los movimientos en pro de la tecnología intermedia o adecuada han ganado adeptos en muchas partes del mundo, pero llevan en su contra el cálculo financiero a nivel de empresa hecho en el marco tradicional microeconómico, la tradición ingenieril del norte extendida a todo el mundo, la indiferencia de gran parte de los

7 Véase, por ejemplo, Gerard K. Boon, "La cambiante ventaja comparativa en el comercio internacional”, Diálogos, núm. 113, vol. 19, México, El Colegio de México, septiembre-octubre de 1983. 
organismos de los países del Tercer Mundo encargados de promover la ciencia y la tecnología, y los prejuicios de muchas profesiones, entre ellas la de los economistas. No parece probable que, en cuanto al criterio de empleo, las tecnologías intermedias lleguen muy lejos, no obstante ejemplos aleccionadores, pero concretados a experiencias poco usuales en muchos países de Asia y África, asi como en varios de América Latina.

La tecnología, en consecuencia, ofrece poco estímulo al empleo masivo de los grandes contingentes de fuerza de trabajo disponible que se avecinan en los países en vía de desarrollo. En los ya desarrollados, en cada restructuración industrial modernizadora, la tecnología desplaza también personal obrero en fuertes volúmenes. Ello induce a comentar la perspectiva particular de los países industrializados del norte en lo que concierne a empleo. En su mayoría, por desajustes estructurales de larga duración, por efecto de la reciente depresión internacional y por el impacto de los sistemas de seguridad social imperantes, el empleo en esos países demuestra escaso dinamismo. Aunque creciera a mediano plazo, no se equilibra con los incrementos, por modestos que sean, de la fuerza de trabajo. Ello se ha manifestado en el creciente rechazo de la mano de obra inmigrada de los países del sur -salvo en mercados de trabajo fragmentados en que ésta puede llevar a cabo, a salarios mínimos, tareas que la mano de obra local no está dispuesta a realizar. Se refleja también en la persistencia de tasas de desempleo muy elevadas, que en algunos casos llegan a cerca del $20 \%$ y parecen situarse en un nivel irreducible de 7 a 12 por ciento (salvo Japón y Suiza).

A ello contribuye la creciente preocupación por lo que ha dado en llamarse la "crisis de los incentivos", sobre todo en las economías de mercado que descansan mayormente en inversión privada para crear nuevas fuentes de empleo. La falta de incentivos puede deberse a tasas tributarias marginales sumamente elevadas, a desconfianza en la estabilidad política, a escaladas de costos antes no internalizados como los de protección ecológica, a los elevados costos de la innovación tecnológica o a la rigidez que crean los beneficios de la seguridad social en la dinámica tradicional de los mercados de trabajo. Debe añadirse el deterioro de los sistemas educativos y de formación, o al menos, en la mayoría de los países industrializados, un desajuste estructural entre los sistemas de enseñanza y formación y las demandas cambiantes del mercado de trabajo. Toda reforma a estos sistemas tropieza con obstáculos y costos mayores, en cualquier régimen político.

Uno de los resultados de toda esta situación es una creciente 
dependencia -y desfinanciamiento - de los aparatos de seguridad social, que en esos países comprenden la protección contra el desempleo; pues en un país de alta industrialización casi nadie puede quedar desprotegido o sin algún ingreso aunque sea en forma de subsidio. Otro resultado es el impacto de los costos de la seguridad social en los costos industriales y de distribución, apenas contrarrestado en parte por las innovaciones de la microelectrónica que, en todo caso, originan desempleo a nivel de unidad de producción. Un tercer resultado es la proliferación de la economía subterránea o informal - una especie de vuelta al subdesarrollo que prospera a base de salarios inframínimos e irregulares, incertidumbre en las condiciones de trabajo y evasión fiscal. Las indemnizaciones por desempleo que recibe un trabajador con frecuencia coinciden con ingresos que obtiene de origen subterráneo, pero a veces éstos son los únicos, sobre todo para los jóvenes entrantes por primera vez a la fuerza de trabajo.

Queda, pues, la duda de que en la perspectiva de aquí al año 2000 los países desarrollados puedan crear empleo suficiente o puedan reducir sus tasas de desempleo abierto. Es más, se examina a nivel profesional, de más en más, una previsión de modalidades de empleo en que se reduciría la semana de trabajo, se alternarían días de trabajo con días de descanso u ocio, o periodos de empleo con periodos de reeducación, reciclaje $o$, de plano, reflexión campestre o urbana sin obligación de trabajo.8

Y todo lo anterior en los países que, según las proyecciones demográficas y de fuerza de trabajo, una vez que pasen el difícil umbral del año 2000 defendiéndose como puedan de la inmigración sureña indocumentada de trabajadores, tendrán, por envejecimiento agudo de su población y dinámica demográfica casi negativa, menos trabajadores propios en edad productiva de lo que pudiera requerir la buena marcha de sus sistemas económicos.

Hagamos ahora un intento de imaginar lo que pudiera ser la perspectiva en los países en vía de desarrollo. A su dinámica poblacional claramente definida se añaden el impacto de la tecnología ahorradora de mano de obra y el creciente costo de la seguridad social. Esta última, si bien raramente se traduce en indemnizaciones

8 Véase por ejemplo, Louis Emmerij, "The Social Economy of Today's Employment Problem in the Industrial Countries", en Edmond Malinvaud y Jean-Paul Fitoussi (compiladores), Unemployment in Western Countries -Proceedings of a Conference Held by the International Economic Association at Bischenberg, France, Londres, MacMillan, 1980, pp. 56-69. 
por desempleo, encuentra compensaciones en el empleo diluido, sobre todo en el sector público. El tradicional subempleo -como quiera que se defina- que en muchos casos abarca del 35 al 45 por ciento de la población económicamente activa y representa ingresos inferiores a los mínimos, ingresos irregulares, falta de protección social y, en los más de los casos, baja productividad física, parece estarse entronizando junto con la marginalidad urbana y la rural -que es la otra cara del mismo fenómeno- que caracteriza desde hace tiempo a la mayoría de los países en desarrollo, sobre todo aquellos en que la dinámica demográfica ha sido, o es todavía, bastante fuerte.

En este punto quisiera asociar el largo plazo del desarrollo con el que llamo el "eterno corto plazo", que hoy como ayer vivimos en la mayoría de los países que componen la economía internacional. No cabe duda alguna que el excesivo endeudamiento del Tercer Mundo, combinado con la depresión internacional, la debilidad de los mercados de productos básicos y el creciente proteccionismo de los países industrializados, plantea un conjunto de factores poco estimulantes para el empleo en esta categoría de países. Es natural que se tenga que concentrar la atención en la restructuración de la deuda externa y en políticas de reordenamiento económico, en la mayoría de los países en desarrollo. Reducir la inflación y cerrar la brecha del sector público son, como tantas veces lo señalara el doctor Opie en sus escritos, acciones indispensables. La alternativa sería el caos. La experiencia está demostrando que el reordenamiento es una tarea mucho más difícil de lo que muchas instituciones monetarias y financieras, nacionales e internacionales, habían pensado, pues ni la inflación es un fenómeno estrictamente monetario, ni se pueden hacer a un lado las presiones sociales y políticas. (En estas materias el doctor Opie y yo solíamos diferir, siempre cordialmente.)

Por otro lado, la economía mundial, si bien no está preparada aún para ajustar y hacer compatibles los diversos desequilibrios estructurales entre el norte y el sur, y aun entre los países del norte, tiene que establecer bases y principios, como en Bretton Woods hace cuarenta años, para que las transacciones financieras y la economía monetaria internacional funcionen orgánicamente. La fuerte sacudida al sistema financiero internacional originada en el excesivo endeudamiento de una gran parte de los países del sur, así como del norte y del este, con fuentes bancarias de recursos financieros no integradas a normas internacionales convenidas entre los gobiernos, ha obligado a acentuar la atención al corto plazo. Y es lógico: sin cortos plazos no es posible llegar a los medianos y a los largos. Y como lo 
expresó en los años treinta la sabiduría de Keynes, "a largo plazo todos estamos muertos". Sin embargo, esa misma sabiduría de él y de sus asesores, previó en Bretton Woods la expansión de las necesidades más lejanas de la economía mundial -aun antes, en el célebre Plan Keynes, pero en todo caso, en forma más limitada, en el Plan White para crear lo que hoy se llama el Banco Mundial, al que han seguido varios bancos regionales como el BID y otros.

Para ser más concreto, preveo hoy tres grandes necesidades: primera, la adopción de medidas de cooperación internacional eficaces para impedir la excesiva inestabilidad de las que el profesor John H. Williams denominó en 1943 las "monedas clave" -que hoy serían unas cuantas más de las tres que él consideraba -;9 segunda, la ampliación de los recursos que componen el sistema del Banco Mundial como apoyo a las necesidades a mediano y a largo plazos del desarrollo (y su complemento descentralizado en los bancos regionales y subregionales); en tercera instancia, la reanudación e intensificación de las cooperaciones económica y financiera internacionales como complemento de los esfuerzos internos de los países en desarrollo. El informe Brandt, y el segundo memorándum de la Comisión del mismo nombre, así como diversas iniciativas en las Naciones Unidas y en varias regiones del Tercer Mundo, apuntan en esta dirección.

No obstante, y a pesar de la posibilidad apenas mediocre de que ello ocurra, se requiere ir más a fondo, más allá de la problemática financiera de corto plazo, se requerirá levantar el velo financiero que cubre a las incongruencias estructurales, para idear, en el mundo altamente interdependiente de hoy y del futuro, una sociedad internacional que en lugar de abolir el empleo lo pueda crear. A falta de ello, iremos hacia un mundo sin empleo, resultado paradójico de la tecnología, de la seguridad social y de la dinámica demográfica.

Para evitar quedarme en generalidades como las que he expresado, creo que mi deber es reflexionar sobre algunos aspectos de la perspectiva del empleo en México. Hace diez años, la preocupación por la excesiva dinámica de la población de México se concretó en una política de población tendiente a reducir la fecundidad, en respuesta a una evidente demanda social a nivel familiar y a una previsión del desarrollo futuro. Sin embargo, este proceso se inició con diez años de retraso; debió haber empezado en los años sesenta, cuando era ya

9 John H. Williams, "La estabilización monetaria: los planes de Keynes y White”, El Trimestre Económico, vol. X, pp. 431-449, México, Fondo de Cultura Económica, 1943. 
evidente la consecuencia de la elevada fecundidad prevaleciente en México, que se traduciría en una fuerza de trabajo cuya tasa anual de incremento es todavía hoy, por la llamada inercia de los procesos demográficos, superior a la del crecimiento general de la población. 10 En los años setenta, ya iniciado el descenso de la fecundidad en México, economistas mexicanos de prestigio nos hicieron saber que si la economía mexicana pudiera crecer al $8 \%$, se absorbería en forma de empleo productivo el incremento anual de la fuerza de trabajo. Aparte de la imposibilidad de mantener por largos años una tasa constante de crecimiento del producto bruto del $8 \%$, la realidad fue que en ningun momento se redujo el desempleo abierto, aun en los años de mayor auge, a menos del 5\% de la PEA, ni se eliminó, pese a la escasez de personal en mercados fragmentados, el subempleo generalizado.

Hoy, con desempleo abierto de alrededor del 12\% de la PEA, que pudiera todavía aumentar, y aun con la reducción de la tasa de crecimiento demográfico de $3.5 \%$ anual a 2.2 en sólo diez años (1974-1984), la avalancha de jóvenes entrantes al mercado de trabajo supera las posibilidades de empleo. Ello plantea para el resto de los años ochenta una sobreoferta de mano de obra que será difícil reducir sustancialmente, sobre todo ante la perspectiva de crecimiento de la economía de alrededor del 5\% anual considerada probable en los años 1985 a 1988.

Sin desestimar las metas previstas en el Plan Nacional de Desarrollo de "lograr una tasa media anual de crecimiento del empleo del 3.5 al 4.0 por ciento" - con la ayuda del "uso de tecnologías más apropiadas" y de la "evolución de las actividades intensivas en mano de obra"-,11 parece necesario escudriñar más allá, hacia los años

10 Sobre la problemática demográfica y de empleo en México a futuro véanse: Centro de Estudios Económicos y Demográficos, Dinámica de la po. blación de México, México, El Colegio de México, 1970; Víctor L. Urquidi, "Empleo y explosión demográfica", Demografía y Economía, vol. VIII, núm. 2, El Colegio de México, 1974; Francisco Alba, La población de México: evo. lución y dilemas, México, El Colegio de México, Centro de Estudios Económicos y Demográficos, 1977, 1984; Benito Roitman, "El empleo en México hacia 1988: proyecciones y perspectivas", Investigación Económica, vol. XIII, núm. 168, México, UNAM, Facultad de Economía, abril-junio de 1984, pp. 231-256; Francisco Javier Alejo, "Empleo y asignación de recursos. México: ¿caso fallido?", en Víctor L. Urquidi y Saúl Trejo (compiladores), Recursos humanos, empleo y desarrollo en la América Latina, México, Fondo de Cultura Económica, Lecturas 51, tomo II, 1983, pp. 140-153.

11 México, Poder Ejecutivo Federal, Plan Nacional de Desarrollo, 1983 
noventa y hacia fin de siglo. Si la proyección de nuestra población total en el año 2000 se cumple a nivel de unos 105 millones de habitantes, será necesario dar para entonces empleo a cerca de 40 millones en actividades productivas, lo que ya representa una tasa de participación considerablemente superior a la actual de $28 \%$. Dado que la fuerza de trabajo de los años noventa estará influida por las tasas de natalidad de los años setenta, es decir, antes de que éstas descendieran en forma pronunciada, la sobreoferta de fuerza de trabajo continuará haciéndose sentir, aunque progresivamente menos a medida que se avance hacia el final del siglo (salvo por mayor participación de la mano de obra femenina en la PEA).

Lo que deseo subrayar es que aun suponiendo en el último decenio del siglo que la economía mexicana creciera a tasa mayor de lo que se prevé en los próximos seis años, el desafío que representa el empleo no podrá resolverse por medio del análisis convencional del fenómeno. El caso de México no es sino ilustrativo de la problemática de la mayor parte de los países en desarrollo, donde la aplicación de la tecnología moderna de avanzada contrarrestará los buenos deseos de instaurar tecnologías y actividades intensivas en mano de obra.

Estos casos particulares no difieren de la perspectiva de un mundo sin empleo, o al menos la de un mundo en que el empleo en los países en desarrollo, el formal y el informal, el subempleo en sus diversas formas, el desempleo disfrazado o encubierto por la proliferación burocrática, tanto pública como privada, y las modalidades de alternación de empleo con educación, reciclaje, descanso y ocio, llevarán a extraordinarias coincidencias con los paises desarrollados, apenas mitigadas por la migración internacional, transitoria o permanente, de la fuerza de trabajo.

Así como en ocasión de la reciente Conferencia Internacional de Población algunos observadores preguntaban si tendría límite el crecimiento poblacional de la zona metropolitana de la ciudad de México o si dicho incremento pudiera ser reversible, hay también quienes se preguntan si las tendencias al desempleo y al subempleo pudieran evitarse en los próximos cuarenta años.

La respuesta a lo primero es que es probable que la zona metropolitana siga creciendo menos rápidamente y que se integrará con el sistema de ciudades circunvecino, a la vez que se adoptarán nuevas formas de circulación, comunicación, asentamiento y convivencia.

1988, México, Secretaría de Programación y Presupuesto, mayo de 1983, p. 211. 
La respuesta a lo segundo pudiera ser que el empleo, como hoy lo entendemos, seguirá encogiéndose en su alcance, y que, sin llegar al "pleno desempleo" o desempleo total que sería un contrasentido, se encontrarán nuevas modalidades, que a su tiempo se institucionalizarán, de utilización del esfuerzo productivo de la fuerza de trabajo, en un marco de responsabilidad social hacia el desempleado o el escasamente empleado.

Pero será un mundo, tanto en el norte como en el sur, que vivirá, con todas sus consecuencias, bajo el síndrome del fin del empleo. 